\title{
Gabapentin for phantom limb pain after amputation in pediatric oncology: a systematic review protocol
}

\author{
Shuang Jiang ${ }^{1}$, Meng-meng Zhou ${ }^{2}$, Rong Xia ${ }^{1}$, Jing-hui Bai ${ }^{3}$ and Li-hui Yan ${ }^{4 *}$
}

\begin{abstract}
Background: Phantom limb pain (PLP) is a prevalent problem for children after amputation because of the chemotherapy treatment. Gabapentin is a potential option to manage PLP after amputation in pediatric oncology. However, no systematic review specifically investigated this topic. Thus, this study aims to appraise the efficacy and safety of gabapentin for post-amputation PLP in pediatric oncology.

Methods: Electronic databases (Cochrane Library, MEDLINE, EMBASE, Web of Science, CINAHL, PsychINFO, Scopus, WANGFANG, and Chinese Biomedical Literature Database) will be systematically searched from the beginning to the present without limitations to publication status and language. Primary outcome is pain intensity. Secondary outcomes are analgesic drug consumption, sleep quality, depression, anxiety, health-related quality of life, and adverse events. The treatment effect of all dichotomous outcome data will be estimated as risk ratio and 95\% confidence intervals (Cls) and that of continuous outcome data will be calculated as mean difference or standardized mean difference and $95 \%$ Cls. Methodological quality of randomized controlled trials (RCTs) will be assessed using Cochrane risk of bias tool and that of case-controlled studies (CCSs) will be appraised using Newcastle-Ottawa Tool. Statistical analysis will be conducted using RevMan 5.3 software.

Discussion: This study will summarize up-to-date high-quality RCTs and CCSs to assess the efficacy and safety of gabapentin for PLP after amputation in pediatric oncology. The findings of this study will help to determine whether or not gabapentin is effective and safe for children with PLP after amputation.
\end{abstract}

Systematic review registration: INPLASY202060090

Keywords: Phantom limb pain, Amputation, Gabapentin, Efficacy, Safety

\section{Background}

Phantom limb pain (PLP) is clinically defined as the perception of pain or discomfort in a missing or amputated limb $[1,2]$. Its symptoms vary from sharp to tingling [3, 4]. PLP in children usually occurs few days in the afternoon or evening, daily, or weekly after limb amputation and typically lasts from seconds to minutes $[5,6]$. In pediatric

\footnotetext{
* Correspondence: yanlh2005@sina.com

${ }^{4}$ Department of Internal Medicine (Pain Clinic), Cancer Hospital of China

Medical University, No. 44 Xiaoheyan Road, Dadong District, Shenyang 110042, China

Full list of author information is available at the end of the article
}

population, the most common causes of PLP are vascular etiologies, trauma, cancer/malignancy, and congenital conditions [6]. It has been estimated that its prevalence rate ranges between 12 and $83 \%$, based on different study reports [7-12]. In the pediatric oncology population, its prevalence rate varies from 48 to $90 \%[7,10,13,14]$. Previous studies have shown that PLP can lead to many problems, including depression, anxiety, and stress, which significantly decreases the health-related quality of life in such patients [9, 15-17]. Currently, its pathophysiology is complex and its mechanisms are still poorly understood.

\section{$\triangle B M C$}

(c) The Author(s). 2021 Open Access This article is licensed under a Creative Commons Attribution 4.0 International License, which permits use, sharing, adaptation, distribution and reproduction in any medium or format, as long as you give appropriate credit to the original author(s) and the source, provide a link to the Creative Commons licence, and indicate if changes were made. The images or other third party material in this article are included in the article's Creative Commons licence, unless indicated otherwise in a credit line to the material. If material is not included in the article's Creative Commons licence and your intended use is not permitted by statutory regulation or exceeds the permitted use, you will need to obtain permission directly from the copyright holder. To view a copy of this licence, visit http://creativecommons.org/licenses/by/4.0/ The Creative Commons Public Domain Dedication waiver (http://creativecommons.org/publicdomain/zero/1.0/) applies to the data made available in this article, unless otherwise stated in a credit line to the data. 
For pediatric oncology patients, amputation is the most commonly utilized management for tumor control in osteosarcoma bone cancer $[18,19]$. It has been reported that the administration of chemotherapy before amputation surgery is one of the most risk factors to develop PLP [7]. Currently, no curative treatments for PLP are available, and its therapy mainly focuses on symptomatic control $[7,20]$. The most common drug for PLP is morphine [21]. However, it can only relieve PLP in about $50 \%$ of patients, and there are still about $30 \%$ of cases having poor response to morphine [22]. Thus, alternative therapy is urgently needed.

Gabapentin, also known as an anticonvulsant or antiepileptic drug, is a structural analog of gammaaminobutyric acid (GABA) agonist, which is utilized for the treatment of epilepsy [23, 24]. It is also used for a variety of neuropathic pain conditions management with an analgesic effect [25]. Studies have suggested that gabapentin has been shown to be effective in several certain types of pain, such as PLP [26-28]. However, its mechanism of action has not been fully explored. Proposed mechanism on the action of gabapentin may enhance GABA release, which can employ an inhibitory effect on pain neurotransmission [29]. Several studies reported that gabapentin can effectively treat PLP after amputation in pediatric oncology [30-33]. However, there is no systematic review that specifically addresses this topic. Thus, this systematic review seeks to provide a comprehensive and systematic review of the best current evidence regarding gabapentin for PLP after amputation in pediatric oncology.

\section{Objective}

The aim of this review is to systematically appraise the evidence of gabapentin for PLP after amputation in pediatric oncology.

This study sought to answer the following questions:

1. Does gabapentin relieve PLP after amputation in pediatric oncology?

2. Is gabapentin safe for the treatment of PLP after amputation in pediatric oncology?

\section{Methods and analysis}

This study has been registered on International Platform of Registered Systematic Review and Meta-analysis Protocols with registration number of INPLASY202060090 (https://www.doi.org;DOI:10.37766/inplasy2020.6.0090). It is designed according to the Guidelines of Preferred Reporting Items for Systematic Reviews and MetaAnalysis (PRISMA) Protocol Statement (Additional file 1) $[34,35]$. Any modifications will be reported in the protocol during the systematic review performance.

\section{Search strategy}

We will systematically retrieve electronic databases (Cochrane Library, MEDLINE, EMBASE, Web of Science, CINAHL, PsychINFO, Scopus, WANGFANG, and Chinese Biomedical Literature Database) from the inception to the present without restrictions to publication status and language. The search strategy is built using keywords including "neoplasms," "pain," "cancer pain," "phantom limb," "pain intensity," "neuropathic," "chronic pain," "gabapentin," "anti-epileptic drug," "anticonvulsant," "neurontin," "gralise," "GABA analogs," "random," "allocation," "placebo," "sham," "blind," "control trial," "case-control," "case control," "case-comparison," "casereferent," "clinical trial," "observational study," "study," and "trial." A detailed search strategy for Cochrane Library is presented in Table 1. Similar search strategies for other electronic databases will be adapted and applied. Translations will be performed when necessary in Chinese databases. At the same time, we will examine unpublished and ongoing work in clinical trial registry, conference proceedings, and reference lists of eligible studies. Two independent reviewers (X-R and B-JH) will carry out the whole process of systematic searches. Any disagreements will be solved by discussion with the help of a third reviewer (Y-LH). A consistent decision will be reached after discussion.

\section{Eligibility criteria for study selection Types of studies}

Primary studies including randomized controlled trials (RCTs) and case-controlled studies (CCSs) will be included. Eligible studies are those that are disseminated up to the present in any language and publication status and those that report one of the outcomes of interest. However, we will exclude animal study, review, editorial letter, case report, case series, non-clinical trial, and uncontrolled study.

\section{Types of participants}

We will include studies involving pediatric oncology patients (under 18 years old) with confirmed bone cancers, irrespective of race, sex, and duration of PLP. In addition, eligible participants are those who suffered from PLP after amputation in pediatric oncology. However, we will excluded participants with multiple metastases, abnormal renal and hepatic function, and allergy to gabapentin and study drugs. In addition, we will also not consider patients with pain caused by other diseases, except PLP.

\section{Types of interventions}

We will consider studies for inclusion that report outcomes in pediatric oncology patients using any forms of gabapentin. 
Table 1 Search strategy of Cochrane Library database

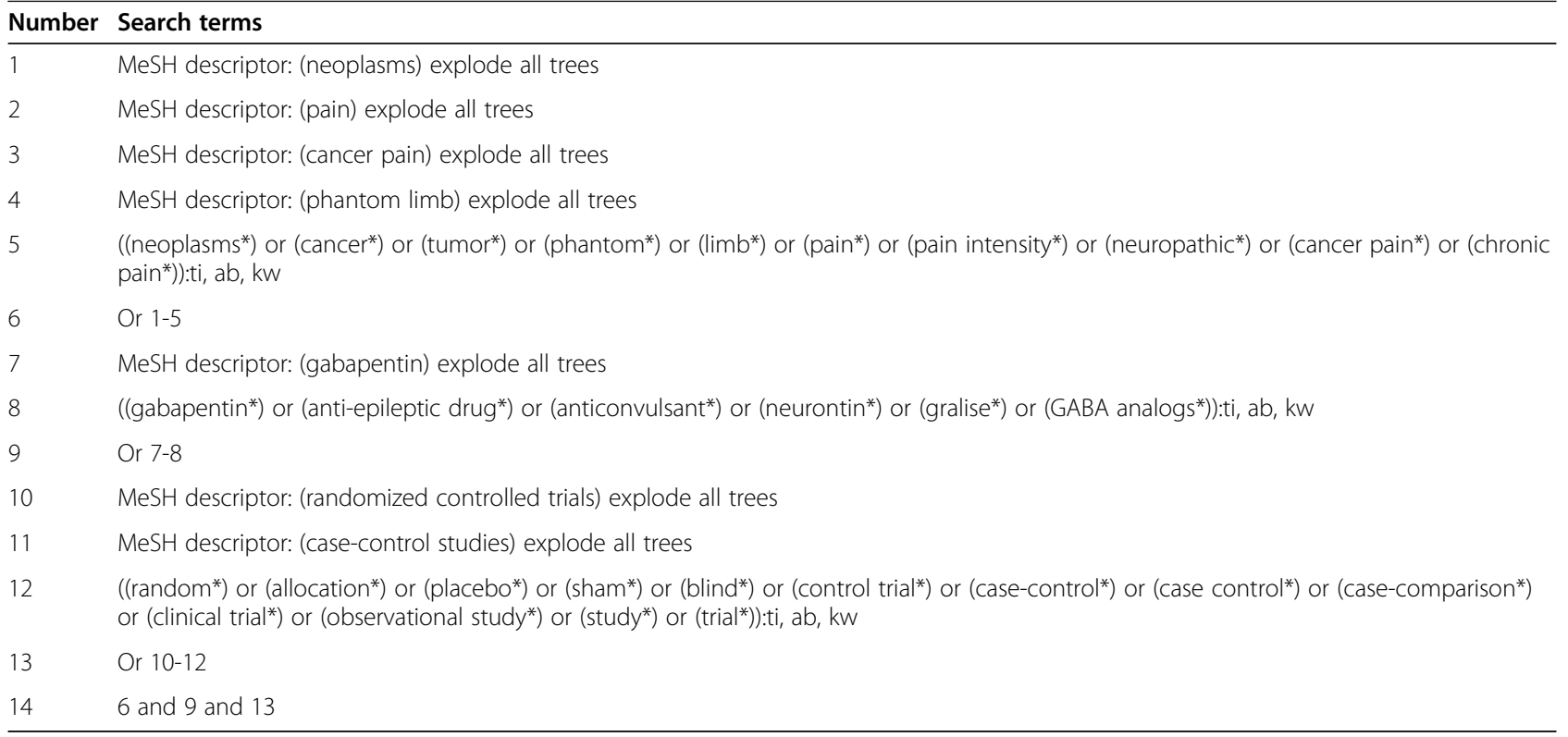

\section{Types of comparators}

There will be no limitations to the comparators, such as routine medication, and placebo. However, we will exclude any comparators that involved any types of gabapentin.

\section{Types of outcomes}

Primary outcome Pain intensity (any pain scale reported in the trial, such as visual analogue scale)

\section{Secondary outcome}

Analgesic drug consumption (any analgesic medication reported in the trial)

Sleep quality (any related scale reported in the trial, such as Medical Outcomes Study Sleep Scale)

Depression (any associated score reported in the trial, such as Zung Depression Scale)

Anxiety (any relevant tool reported in the trial, such as Beck Anxiety Inventory)

Health-related quality of life (any relevant tool reported in the trial, such as 36-Item Short Form Survey)

Adverse events (any records reported in the trial)

\section{Study selection}

Records retrieved from the literature sources will be imported to a reference management, and duplicates will be eliminated. Two independent authors (J-S and ZMM) will carry out a broad scan of study titles and abstracts; and unrelated studies will be removed. Then, full-paper of potential eligible studies will be checked to make sure whether they fulfill all eligibility criteria. Any divergences between two authors will be solved by consulting a third author (X-R), and a final decision will be reached after the discussion. Reasons for all excluded studies at different stages will be recorded. The results of study selection process will be summarized in a PRIS MA flowchart.

\section{Data extraction and management}

Data from selected RCTs and CCSs will be transferred from their original presentation to a standard form with each included study receiving a reference code. If necessary, we will also extract indirect data from figures and charts.

For all included RCTs and CCSs, two authors (J-S and Z-MM) will independently obtain the data from eligible trials according to the predefined data extraction sheet developed specifically for this study. Any opposite views regarding the data extraction will be resolved by discussion with the help of another author $(X-R)$, and we will make a final consistent decision. The extracted information consists of study characteristics (such as country, title, language, publication time, and funding source), patient characteristics (such as age, gender, and diagnostic criteria), study design (such as randomization details, blind, and lost to follow-up), intervention and control details (such as treatment types, duration, and number and length of sessions), and outcomes, safety, and other related information (such as confounding factors).

\section{Study quality assessment}

The study quality of each eligible study will be examined by two independent authors (X-R and B-JH) using Cochrane Collaboration's Risk of Bias Tool for RCTs [36] and Newcastle-Ottawa Scale for CCSs [37], with 
predetermined criteria. RCTs will be assessed on seven aspects, and each one is further rated as high, unclear, or low risk of bias [36]. CCSs will be appraised on three broad perspectives with eight specific items [37]. Any doubt between two authors will be answered with the help of a third author (Y-LH) through discussion, and a final consistent decision will be made.

\section{Dealing with missing data}

We will contact corresponding authors of primary studies to obtain any missing or insufficient or unclear data by email or fax. If we can not achieve those data, we will analyze available data using Intention-To-Treat approach and will discuss its potential affects in the manuscript.

\section{Assessment of reporting bias}

If there is a minimum of 10 trials in any meta-analysis, we will examine reporting bias using a funnel plot [38], and symmetry of the funnel plot will be performed using Egger's regression test [39].

\section{Data synthesis}

We will use RevMan 5.3 software to synthesize and analyze all outcome data. We plan to carry out separate analysis based on types of study, including RCTs and CCSs. We will calculate the treatment effect of dichotomous data using risk ratio and $95 \%$ confidence intervals (CIs) and that of continuous data using mean difference (MD) or standardized MD and 95\% CIs. We will examine heterogeneity using $I^{2}$ statistic, and we will undertake statistical pooling on groups of trials which are considered to be sufficiently similar $[40,41]$. Where heterogeneity is low or minor $\left(I^{2} \leq 25 \%\right)$, we will utilize a fixed-effect model to pool the data; if heterogeneity is moderate $\left(25 \%<I^{2} \leq 75 \%\right)$, we will apply a random-effect model to synthesize the data, and if heterogeneity is obvious $\left(I^{2}>75 \%\right)$, we will not pool the data [40]. Meta-analysis will be carried out based on the sufficient homogeneity regarding on participant characteristics, types of intervention and outcome, and comparability between methods and ability to aggregate data. A narrative synthesis of eligible trials will be performed if the extracted data is too diverse to fulfill the threshold for meta-analytic approach. We will build a "summary of findings" table for the outcomes, and we will appraise evidence quality of primary outcome using Grading of Recommendations Assessment, Development and Evaluation [42, 43], which covers five aspects of risk of bias, imprecision, consistency of effect, indirectness, and publication bias.

\section{Subgroup analysis}

We will carry out subgroup analysis to test the sources of significant heterogeneity based on the following:

Studies at low risk of bias compared to high risk of bias Studies stratified according to different forms of gabapentin, such as single modality and combined managements

Studies stratified based on the control treatments Studies stratified in accordance with the different geographical regions, and outcomes at different time points

\section{Sensitivity analysis}

We will investigate the sensitivity analysis to test the stability and robustness of study findings based on the sample size of included trials, and study quality.

\section{Dissemination}

We will publish this study on a peer-reviewed journal or a conference meeting.

\section{Discussion}

This systematic review will allow us to separately synthesize the findings of RCTs and CCSs addressing the efficacy and safety of gabapentin for PLP after amputation in pediatric oncology. It will be based on the eligible published studies from the inception to the present and will allow us to assess study quality and analyze outcome data. It will also provide associated information on current knowledge of gabapentin for PLP after amputation in pediatric oncology. This will be conducted by accessing information without publication status and language limitations.

A variety of clinical trials suggested that gabapentin can relieve PLP after amputation in pediatric oncology. However, no systematic review is identified on investigating the efficacy and safety of gabapentin for PLP after amputation in children population comprehensively. Thus, this study represents the first systematic review to examine the efficacy and safety of gabapentin for PLP after amputation in children with oncology. We expect our results that should allow us to draw beneficial conclusions about the efficacy and safety of gabapentin for PLP after amputation in pediatric oncology, which may benefit both clinicians and future studies.

\section{Supplementary Information}

The online version contains supplementary material available at https://doi. org/10.1186/s13643-020-01571-8.

Additional file 1. PRISMA-P Checklist.

\section{Abbreviations}

PLP: Phantom limb pain; GABA: Gamma-aminobutyric acid; PRISMA: Preferred Reporting Items for Systematic Reviews and Meta-Analysis; 
RCTs: Randomized controlled trials; CCSs: Case-controlled studies;

Cls: Confidence intervals; MD: Mean difference

\section{Acknowledgements}

Not applicable.

\section{Authors' contributions}

SJ and LHY conceived the study. RX and JHB contributed their expertise and to the clinical background. MMZ and RX contributed to the analytical plan and the bias assessment approach. SJ, JHB, and LHY performed the literature search plan and drafted the protocol. All authors revised the protocol and approved the final version. LHY supervised the study.

\section{Funding}

Not applicable.

\section{Availability of data and materials}

Data sharing is not applicable to this article as no datasets were generated or analyzed during the current protocol.

\section{Ethics approval and consent to participate}

Not applicable.

\section{Consent for publication}

Not applicable.

\section{Competing interests}

The authors declare that they do not have competing interests.

\section{Author details}

${ }^{1}$ Department of Internal Medicine (Psychologic Clinic), Cancer Hospital of China Medical University, Shenyang 110042, China. ${ }^{2}$ Department of Psychology Clinic, Shengjing Hospital of China Medical University, Shenyang 110022, China. ${ }^{3}$ Department of Internal Medicine (VIP Ward), Cancer Hospital of China Medical University, Shenyang 110042, China. ${ }^{4}$ Department of Internal Medicine (Pain Clinic), Cancer Hospital of China Medical University, No. 44 Xiaoheyan Road, Dadong District, Shenyang 110042, China.

Received: 23 June 2020 Accepted: 22 December 2020

Published online: 13 January 2021

\section{References}

1. Hsu E, Cohen SP. Postamputation pain: epidemiology, mechanisms, and treatment. J Pain Res. 2013:6:121-36.

2. Probstner D, Thuler LC, Ishikawa NM, Alvarenga RM. Phantom limb phenomena in cancer amputees. Pain Pract. 2010;10(3):249-56.

3. Schley MT, Wilms P, Toepfner S, Schaller HP, Schmelz M, Konrad CJ, et al. Painful and nonpainful phantom and stump sensations in acute traumatic amputees. J Trauma. 2008;65(4):858-64.

4. Ehde DM, Czerniecki JM, Smith DG, Campbell KM, Edwards WT, Jensen MP et al. Chronic phantom sensations, phantom pain, residual limb pain, and other regional pain after lower limb amputation. Arch Phys Med Rehabil. 2000;81:1039-44

5. Wilkins KL, McGrath PJ, Finley GA, Katz J. Phantom limb sensations and phantom limb pain in child and adolescent amputees. Pain. 1998;78:7-12.

6. Wilkins KL, McGrath PJ, Finley GA, Katz J. Prospective diary study of nonpainful and painful phantom sensations in a preselected sample of child and adolescent amputees reporting phantom limbs. Clin J Pain. 2004; 20(5):293-301.

7. Smith J, Thompson JM. Phantom limb pain and chemotherapy in pediatric amputees. Mayo Clin Proc. 1995;70:357-64.

8. Thomas CR, Brazeal BA, Rosenberg L, Robert RS, Blakeney PE, Meyer WJ. Phantom limb pain in pediatric burn survivors. Burns. 2003:29:139-42.

9. Ephraim PL, Wegener ST, MacKenzie EJ, Dillingham TR, Pezzin LE. Phantom pain, residual limb pain, and back pain in amputees: results of a national survey. Arch Phys Med Rehabil. 2005;86(10):1910-9.

10. Poor Zamany Nejatkermany M, Modirian E, Soroush M, Masoumi M, Hosseini M. Phantom limb sensation (PLS) and phantom limb pain (PLP) among young landmine amputees. Iran J Child Neurol. 2016;10:42-7.

11. Richardson C, Glenn S, Nurmikko T, Horgan M. Incidence of phantom phenomena including phantom limb pain 6 months after major lower limb amputation in patients with peripheral vascular disease. Clin J Pain. 2006; 22(4):353-8.

12. Flor H. Phantom-limb pain: characteristics, causes, and treatment. Lancet Neurol. 2002;1:182-9.

13. Burgoyne LL, Billups CA, Jirón JL Jr, Kaddoum RN, Wright BB, Bikhazi GB, et al. Phantom limb pain in young cancer-related amputees: recent experience at St Jude children's research hospital. Clin J Pain. 2012;28(3): 222-5.

14. Anghelescu DL, Kelly CN, Steen BD, Wu J, Wu H, DeFeo BM, et al. Mirror therapy for phantom limb pain at a pediatric oncology institution. Rehabil Oncol. 2016;34(3):104-10.

15. van der Schans CP, Geertzen JH, Schoppen T, Dijkstra PU. Phantom pain and health-related quality of life in lower limb amputees. J Pain Symptom Manage. 2002;24:429-36.

16. Desmond DM, MacLachlan M. Prevalence and characteristics of phantom limb pain and residual limb pain in the long term after upper limb amputation. Int J Rehabil Res. 2010:33:279-82.

17. Arena JG, Sherman RA, Bruno GM, Smith JD. The relationship between situational stress and phantom limb pain: cross-lagged correlational data from six month pain logs. J Psychosom Res. 1990;34:71-7.

18. Han G, Bi WZ, Xu M, Jia JP, Wang Y. Amputation versus limb-salvage surgery in patients with osteosarcoma: a meta-analysis. World J Surg. 2016;40(8):2016-27.

19. MacEwen EG, Kurzman ID. Canine osteosarcoma: amputation and chemoimmunotherapy. Vet Clin North Am Small Anim Pract. 1996;26(1): 123-33.

20. Link M, Gebhardt M, Meyers P. Osteosarcoma. In: Pizzo PA, Poplack DG, editors. Principles and practices of pediatric oncology. Philadelphia: Lippincott Williams \&Wilkins; 2006. p. 1074-115.

21. Wu CL, Agarwal S, Tella PK, et al. Morphine versus mexiletine for treatment of postamputation pain: a randomized, placebo-controlled, crossover trial. Anesthesiology. 2008;109:289-96.

22. Nikolajsen $L$, Jensen TS. Phantom limb pain. Br J Anaesth. 2001:87:107-16.

23. Andrews $\mathrm{CO}$, Fischer JH. Gabapentin: a new agent for the management of epilepsy. Ann Pharmacother. 1994;28(10):1188-96.

24. Goa KL, Sorkin EM. Gabapentin. A review of its pharmacological properties and clinical potential in epilepsy. Drugs. 1993;46(3):409-27.

25. Rosenberg JM, Harrell C, Ristic H, Werner RA, deRosayro M. The effect of gabapentin on neuropathic pain. Clin J Pain. 1997;13:251-5.

26. Samineni VK, Premkumar LS, Faingold CL. Neuropathic pain-induced enhancement of spontaneous and pain-evoked neuronal activity in the periaqueductal gray that is attenuated by gabapentin. Pain. 2017;158(7): 1241-53.

27. Rimaz S, Alavi CE, Sedighinejad A, Tolouie M, Kavoosi S, Koochakinejad L. Effect of gabapentin on morphine consumption and pain after surgical debridement of burn wounds: a double-blind randomized clinical trial study. Arch Trauma Res. 2012;1(1):38-43.

28. Abbass K. Efficacy of gabapentin for treatment of adults with phantom limb pain. Ann Pharmacother. 2012;46(12):1707-11.

29. Doleman B, Heinink TP, Read DJ, et al. A systematic review and metaregression analysis of prophylactic gabapentin for postoperative pain. Anaesthesia. 2015;70:1186-204.

30. Rusy LM, Troshynski TJ, Weisman SJ. Gabapentin in phantom limb pain management in children and young adults: report of seven cases. J Pain Symptom Manage. 2001;21(1):78-82.

31. Bone M, Critchley P, Buggy DJ. Gabapentin in postamputation phantom limb pain: a randomized, double-blind, placebo-controlled, cross-over study. Reg Anesth Pain Med. 2002;27(5):481-6.

32. Wang $X$, Yi Y, Tang D, Chen Y, Jiang Y, Peng J, et al. Gabapentin as an adjuvant therapy for prevention of acute phantom-limb pain in pediatric patients undergoing amputation for malignant bone tumors: a prospective double-blind randomized controlled trial. J Pain Symptom Manage. 2018; 55(3):721-7.

33. Kendall MC, Castro-Alves LJ. Gabapentin to prevent acute phantom-limb pain in pediatric patients undergoing amputation. J Pain Symptom Manage. 2018;56(2):e4

34. Shamseer L, Moher D, Clarke M, et al. PRISMA-P Group. Preferred reporting items for systematic review and meta-analysis protocols (PRISMA-P) 2015: elaboration and explanation. BMJ. 2015;349:g7647.

35. Moher D, Shamseer L, Clarke M, et al. Preferred reporting items for systematic review and meta-analysis protocols (PRISMA-P) 2015 statement. Syst Rev. 2015;4:1 
36. Higgins JPT, Altman DG, Gøtzsche PC, et al. The Cochrane collaboration's tool for assessing risk of bias in randomised trials. BMJ. 2011;343:d5928.

37. Stang A. Critical evaluation of the Newcastle-Ottawa scale for the assessment of the quality of nonrandomized studies in meta-analyses. Eur J Epidemiol. 2010;25(9):603-5.

38. Sutton AJ, Duval SJ, Tweedie RL, et al. Empirical assessment of effect of publication bias on meta-analyses. BMJ. 2000;320:1574-7.

39. Egger M, Davey Smith G, Schneider M, et al. Bias in meta-analysis detected by a simple, graphical test. BMJ. 1997;315:629-34.

40. Higgins JPT, Thompson SG, Deeks JJ, Altman DG. Measuring inconsistencies in meta-analysis. BMJ. 2003;327(7414):557-60.

41. Page MJ, Altman DG, McKenzie JE, Shamseer L, Ahmadzai N, Wolfe D, et al. Flaws in the application and interpretation of statistical analyses in systematic reviews of therapeutic interventions were common: a crosssectional analysis. J Clin Epidemiol. 2018;95:7-18.

42. Guyatt GH, Oxman AD, Vist GE, et al. GRADE: an emerging consensus on rating quality of evidence and strength of recommendations. BMJ. 2008;336:924-6.

43. Atkins $D$, Best $D$, Briss PA, et al. Grading quality of evidence and strength of recommendations. BMJ. 2004;328:1490.

\section{Publisher's Note}

Springer Nature remains neutral with regard to jurisdictional claims in published maps and institutional affiliations.

Ready to submit your research? Choose BMC and benefit from:

- fast, convenient online submission

- thorough peer review by experienced researchers in your field

- rapid publication on acceptance

- support for research data, including large and complex data types

- gold Open Access which fosters wider collaboration and increased citations

- maximum visibility for your research: over $100 \mathrm{M}$ website views per year

At $\mathrm{BMC}$, research is always in progress.

Learn more biomedcentral.com/submissions 